\title{
IMPACTO DE LA FERTILIZACIÓN CARBÓNICA EN LOS INVERNADEROS DEL PONIENTE ALMERIENSE
}

\author{
$\underline{\text { Javier Martínez-Dalmau }}^{\text {a*}}{ }^{*}$ Blanca Cuadrado-Alarcón ${ }^{b}$, Alfonso Expósito $^{\mathrm{c}}$, Julio Berbel $^{\mathrm{d}}$ \\ ${ }^{a}$ Universidad de Córdoba, Campus Rabanales, Ctra. N-IV km 396 (Córdoba, javier.martinez@uco.es). ${ }^{b}$ \\ Instituto de Agricultura Sostenible (IAS) Consejo Superior de Investigaciones Científicas (CSIC), Campus \\ Alameda del Obispo, Avenida Menéndez. Pidal s/n (Córdoba, cuadradoblanca@gmail.com). ${ }^{c}$ WEARE- \\ Water, Environmental and Agricultural Resources Economics Research Group, Departamento de \\ Economía Aplicada, Universidad de Málaga, Ejido 6, (Malaga, aexposito@uma.es). ${ }^{d}$ WEARE-Water, \\ Environmental and Agricultural Resources Economics Research Group, Departamento de Economía \\ Agraria, Universidad de Córdoba, Campus Rabanales, Ctra. N-IV km 396 (Córdoba, berbel@uco.es)
}

\section{Resumen}

El objetivo de esta investigación es proporcionar una evaluación del impacto de la fertilización carbónica (FC) con especial atención a la dimensión económica/empresarial y al agua utilizada (como recurso económico relevante en zonas con escasez de agua) en el caso concreto de los invernaderos de Almería (zona del Campo de Dalías, España). Como se puede observar en la evaluación económica realizada en este estudio, el uso de la FC puede contribuir significativamente a la mejora de la producción, al aumento de la calidad de los cultivos y a la viabilidad empresarial en esta zona agrícola de España.

Palabras clave: Fertilización carbónica, invernaderos, beneficio.

\section{Introducción}

Campo de Dalías es una comarca situada al suroeste de Almería (España) caracterizada por una actividad agrícola intensiva. Con una superficie de 21,285 ha de invernaderos dedicados a la producción de hortalizas (CAPDR, 2017), Campo de Dalías es la zona con mayor densidad de invernaderos de Europa. La agricultura supone el $40 \%$ de la actividad económica de la comarca (Baeza Cano, 2014) y configura una de las zonas de invernaderos más importantes, no sólo de Europa, sino también del mundo, en cuanto a la producción y comercialización de hortalizas de fuera de temporada.

Campo de Dalías, y más genéricamente Almería, se caracteriza por una tecnología local denominada invernadero "tipo Almería" con el subtipo 'raspa y amagado' como el más común (Valera et al., 2017) y su ventaja competitiva fundamental es el menor consumo energético durante el proceso de producción debido a las características climáticas de la zona (Cajamar, 2020; Valera et al., 2017). Sin embargo, en la actualidad, el sector de producción de hortícolas del Campo de Dalías se ve amenazado por sistemas de invernaderos de alta tecnología, como los de Holanda, y por sistemas menos avanzados técnicamente, como los de Marruecos, pero con menores costes de producción (Costa and Heuvelink, 2005). Esto ha provocado que determinados cultivos (i.e., judía verde y tomate) hayan desaparecido de los invernaderos del Campo de Dalías debido a su inviabilidad económica (Aznar-Sánchez et al., 2020).

El valor social y económico de la horticultura en la comarca del Campo de Dalías y su relevancia como zona productiva estratégica en la Unión Europea, configuran la necesidad de evaluar escenarios productivos futuros que incluyan innovaciones técnicas agronómicas alternativas, como la fertilización carbónica (FC). Este estudio pretende ofrecer una evaluación del impacto de la FC con especial atención a la dimensión económica/empresarial en la mayor zona de invernaderos de España (el Campo de Dalías).

La literatura existente se ha centrado en gran medida en el análisis del impacto de la FC en variables agronómicas y en sus impactos ambientales. En cambio, las repercusiones económicas de la FC para el agricultor y el sector agrícola han recibido mucha menos atención. Hasta donde sabemos, no existe ningún estudio que ofrezca una evaluación completa del impacto económico de la FC aplicada en una zona agrícola extensa, como es el caso del Campo de Dalías. El presente estudio pretende subsanar esta laguna.

\section{Metodología}

Los datos publicados sobre el sector de los invernaderos están disponibles a nivel provincial, por lo que los datos a nivel local (Campo de Dalías) tienen que ser estimados a partir de la información disponible. Los datos disponibles sobre la gestión de los cultivos en Almería se han recopilado cuidadosamente para modelar los márgenes netos de cada rotación de cultivos. A continuación, se describen detalladamente las fuentes de información:

a. Superficie de invernadero del Campo de Dalías (ha) (CAPDR, 2017).

b. Combinaciones de cultivos presentes en el Campo de Dalías (Lopez Perez et al., 2015).

c. Rendimiento del cultivo por tipo de invernadero (Kg/ha) (Valera et al., 2017).

d. Costes del cultivo (euros/ha). El Observatorio de Precios y Mercados (OPM, 2014-2019a). 
e. Distribución mensual de los productos comercializados (\%) (OPM, 2014-2019b).

f. Necesidad de agua de los cultivos (m3/ha) (EEFC, 2009; Fernández et al., 2007).

g. Precio mensual del cultivo (EUR/kg).

h. Respuesta de los cultivos a la FC. (Alonso et al., 2010, 2012; Dong et al., 2020; Lorenzo et al., 1990; Sánchez-González et al., 2016; Sánchez-Guerrero, 1999; Villachica-León, 2018).

Además, se han realizado varias entrevistas con profesionales e investigadores expertos en la zona de estudio, para contrastar las variables estimadas con la realidad de la actividad agraria en el Campo de Dalías. Entre los colaboradores más importantes se encuentran: el Instituto de Investigación y Formación Agraria y Pesquera (IFAPA-La Mojonera); la Estación Experimental de Cajamar; la Agencia de Gestión Agraria y Pesquera de Andalucía (AGAPA); la Universidad de Almería; la Asociación Agraria de Jóvenes Agricultores (ASAJA) de Almería; y otros agentes del sector local.

La FC es inviable en los invernaderos de 'raspa y amagado', debido a problemas de estanqueidad. Por lo tanto, para analizar la aplicación de esta innovación tecnológica en Campo de Dalías, es necesario evaluar previamente el impacto de la implantación de invernaderos aptos para aplicar la FC 'multitunel'.

A partir de los datos originales, se ha realizado una estimación del beneficio proporcionado por cada ha cultivada bajo el sistema de invernaderos actualmente instalado en la zona. Estos resultados permiten estimar los parámetros de incremento de costes e ingresos correspondientes al cambio de tecnología, primero del sistema 'raspa y amagado' al de 'multitunel' y posteriormente, de éste al sistema de invernadero 'multitunel $+F C^{\prime}$.

Gráfico 1. Modelización del margen bruto de los invernaderos de "raspa y amagado" (las letras indican la fuente de los datos)

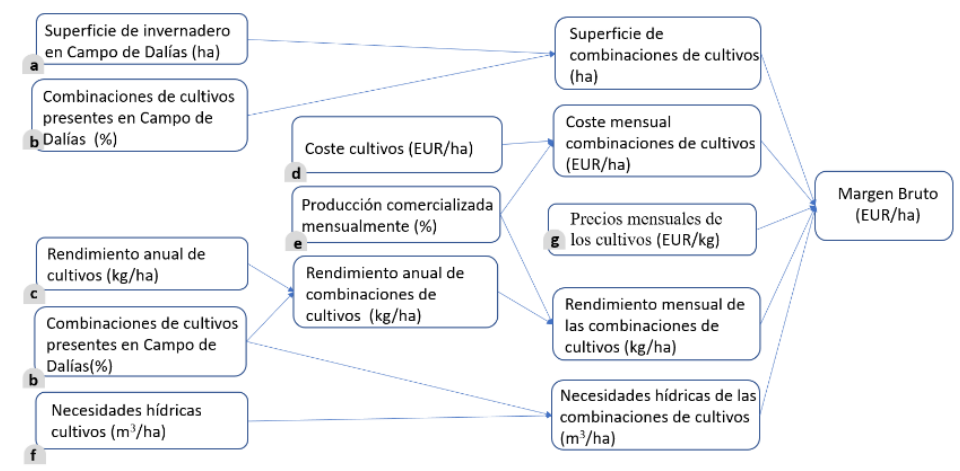

Una vez estimado el margen bruto por ha en la tecnología 'raspa y amagado', los resultados obtenidos por ha se extrapolaron a toda la superficie de invernaderos de la comarca. Se establecen tres escenarios:

- Escenario 0 (situación actual): 95\% de la superficie total de invernaderos con 'raspa y amagado'; 5\% de la superficie total de invernaderos con 'multitunel' (Valera et al., 2017) de los cuales solo el 10\% utiliza CF.

- Escenario 1. Crecimiento en el uso de la FC que se utilizaría en el 100\% de los invernaderos 'multitunel' pero siguiendo el patrón del tipo de invernadero respecto al escenario 0, es decir, 95\% 'raspa y amagado'; $5 \%$ 'multitunel' (todos ellos ya con FC).

- Escenario 2. El 25\% de los invernaderos del Campo de Dalias pasan a ser invernaderos 'multitunel', todos ellos con CF.

Gráfico 2. Esquema general de la estimación de los diferentes escenarios

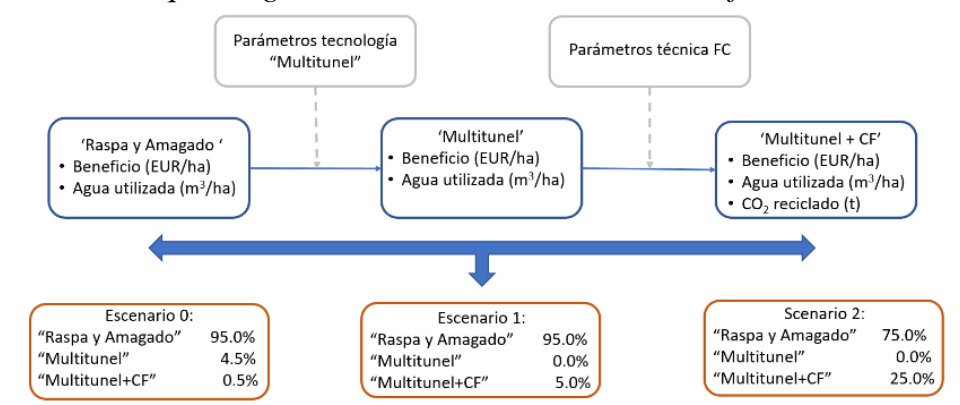

Debido a la incertidumbre sobre el incremento de precio inducido por la $\mathrm{FC}$ en el producto final comercializado, realizamos un análisis de sensibilidad de cada escenario de acuerdo con las siguientes hipótesis: 
a) La FC no induce ningún aumento de precios.

b) La FC induce un aumento de precios del $10 \%$

\section{Resultados}

El cuadro 1 resume los impactos de la FC en términos de beneficio económico, necesidades de mano de obra, $\mathrm{CO}_{2}$ reciclado y uso del agua cuando se aplica a las 21,285 ha cultivadas en Campo de Dalías bajo los escenarios alternativos $(1,2)$ y variación de precios $(\mathrm{a}, \mathrm{b})$ definidos en el epígrafe 2.

Cuadro 1. Resultados de los diferentes escenarios frente al escenario de referencia

\begin{tabular}{lcccc}
\cline { 2 - 4 } & $\begin{array}{c}\Delta \text { Beneficio } \\
(\text { EUR })\end{array}$ & $\begin{array}{c}\Delta \text { Mano de Obra } \\
\text { (jornales) }\end{array}$ & $\begin{array}{c}\Delta \mathrm{CO}_{2} \text { Reciclado } \\
(\mathrm{t} / \mathrm{a})\end{array}$ & $\begin{array}{c}\Delta \text { Agua usada } \\
\left(\mathrm{m}^{3}\right)\end{array}$ \\
\cline { 2 - 5 } Escenario 1a $(\sin \Delta$ precio por FC) & $5,323,060$ & 22,515 & 95,783 & -- \\
\hline Escenario 1b $(\operatorname{con} \Delta$ precio=10\% por FC) & $18,208,578$ & 22,515 & 95,783 & - \\
\hline Escenario 2a $(\sin \Delta$ precio por FC) & $68,092,799$ & 227,702 & 425,700 & $5,673,547$ \\
\hline Escenario 2b $(\operatorname{con} \Delta$ precio=10\% & $132,570,389$ & 227,702 & 425,700 & $5,673,547$
\end{tabular}

Todos los indicadores registran una mejora. En concreto, en el escenario 1a, la rentabilidad económica de la comarca aumenta 5.3 MEUR respecto a la situación de partida (escenario 0), con una creación de 22,515 nuevos puestos de trabajo y 95,783 t de $\mathrm{CO}_{2}$ industrial reciclado. Todo ello sin que se produzca aumento de consumo de agua debido a la reducción de conductancia estomática y de la tasa de transpiración del cultivo provocada por las altas concentraciones de $\mathrm{CO}_{2}$. En el escenario $1 \mathrm{~b}$, que supone un aumento del precio de los cultivos del 10\% debido a la FC, el incremento de la rentabilidad económica asciende a 18,2 MEUR.

Tanto el escenario 2 a como el $2 \mathrm{~b}$ muestran un aumento significativo de los beneficios económicos hasta 68 y 132 MEUR, respectivamente. El aumento de las necesidades de mano de obra es diez veces superior al del escenario 1 y el $\mathrm{CO}_{2}$ reciclado aumenta en 425,700 t/a, en comparación con el escenario de referencia. En este escenario (2), el paso de la tecnología 'raspa y amagado' a la 'multitunel' induce un aumento de la productividad de la tierra, lo que explica el aumento observado de la demanda de agua de $5.6 \mathrm{Mm}^{3}$ frente a la del escenario de referencia.

\section{Conclusión}

Este estudio muestra cómo el uso de $\mathrm{CF}$ en forma de $\mathrm{CO}_{2}$ reciclado capturado del sector industrial (por ejemplo, la generación de energía) puede mejorar la rentabilidad del sector de los invernaderos en la zona del Campo de Dalías. Este resultado está en consonancia con otros estudios, como el de Alonso et al. (2012) y el de Stanghellini et al. (2008) que afirman que el aumento de los ingresos debido a la mayor productividad es mayor que los costes del FC, lo que garantiza un beneficio económico. Sin embargo, no se encuentra la misma unanimidad a la hora de evaluar la aplicación de esta técnica en las condiciones tecnológicas y climáticas de Almería. De Pascale and Maggio (2008) indican que la sostenibilidad de la FC en los invernaderos de Almería está ligada a la aplicación de sistemas de calefacción, que en Almería no se utilizan debido a sus características climáticas. Como se desprende de la evaluación económica realizada en este estudio, el uso de la FC puede contribuir significativamente a mejorar la producción, aumentar la calidad de las cosechas y asegurar la viabilidad del negocio en esta zona agrícola de España. Todos estos beneficios redundan en una mayor rentabilidad de la explotación de los invernaderos, aumentando así su viabilidad empresarial y su competitividad en el mercado.

\section{Referencias}

Alonso, F., Lorenzo, P., Medrano, E., Sánchez-Guerrero, M., 2010. Greenhouse sweet pepper productive response to carbon dioxide enrichment and crop pruning, XXVIII International Horticultural Congress on Science and Horticulture for People (IHC2010): International Symposium on 927. pp. 345351.

Alonso, F., Lorenzo, P., Medrano, E., Sánchez-Guerrero, M., 2012. Evaluación de la técnica de enriquecimiento carbónico en invernadero mediterráneo en un cultivo de pimiento. Actas de Horticultura 60, 348-353.

Aznar-Sánchez, J.A., Velasco-Muñoz, J.F., García-Arca, D., López-Felices, B., 2020. Identification of Opportunities for Applying the Circular Economy to Intensive Agriculture in Almería (South-East Spain). Agronomy 10(10), 1499, doi: https://doi.org/10.3390/agronomy10101499. 
Baeza Cano, R., López Segura, J.G., Dominguez Prats, P., de Miguel Gómez, J., Cánovas Fernandez, G., 2014. Los recursos hídricos en el Campo de Dalías. Consejería de Agricultura, Pesca y Desarrollo Rural. Instituto de Investigación y Formación Agraria y Pesquera Centro IFAPA La Moraleja. Almería.

Cajamar, 2020. Análisis de la campaña hortofrutícola. Campaña 2018/2019. https://infogram.com/analisis-de-la-campana-hortofruticola-1hd12y9wyv3x6km. (Accessed 10 February 2021).

CAPDR, 2017. Cartografia de Invernaderos en Almeria, Granada y Malaga. Año 2017, Report prepared by the Consejeria de Agriculura, Pesca y Desarrollo Rural. Junta de Andalucía, Seville, Spain.

Costa, J., Heuvelink, E., 2005. Introduction: the tomato crop and industry, Tomatoes. CABI Publishing, Wallingford, pp. 1-19.

De Pascale, S., Maggio, A., 2008. PLANT STRESS MANAGEMENT IN SEMIARID GREENHOUSE, 797 ed. International Society for Horticultural Science (ISHS), Leuven, Belgium, pp. 205-215.

Dong, J., Gruda, N., Li, X., Tang, Y., Zhang, P., Duan, Z., 2020. Sustainable vegetable production under changing climate: The impact of elevated $\mathrm{CO} 2$ on yield of vegetables and the interactions with environments-A review. Journal of Cleaner Production 253, 119920, doi: https://doi.org/10.1016/j.jclepro.2019.119920.

EEFC, 2009. Dosis de riego para los cultivos hortícolas bajo invernadero en Almería. Report prepared by the Estación Esperimentál de la Fundación Cajamar, Almería, Spain.

Fernández, M., González, A., Carreño, J., Perez, C., Bonachela, S., 2007. Analysis of on-farm irrigation performance in Mediterranean greenhouses. Agricultural Water Management 89(3), 251-260, doi: https://doi.org/10.1016/j.agwat.2007.02.001.

Lopez Perez, R., Lorbach Kelle, M., Polonio Baeyens, D., Manrique Gordillo, T., 2015. Caracterización de los invernaderos de Andalucía, Junta de Andalucía.

Lorenzo, P., Maroto, C., Castilla, N., 1990. CO2 in plastic greenhouse in Almeria (Spain). Acta Horticulturae(268), 165-169.

OPM, 2014-2019a. Costes de producción por producto. Hortícolas protegidas. https://www.juntadeandalucia.es/agriculturaypesca/observatorio/servlet/FrontController?action=List\&tabl $\mathrm{e}=11210 \&$ page $=1$. (Accessed 25 February 2021).

OPM, 2014-2019b. Datos básicos del producto. Hortícolas protegidas. https://www.juntadeandalucia.es/agriculturaypesca/observatorio/servlet/FrontController?action=List\&tabl e=11114\&page=1. (Accessed 09 March 2021).

Sánchez-González, M.J., Sánchez-Guerrero, M.C., Medrano-Cortés, E.M., Porras, M.E., Baeza, E.J., Lorenzo-Mínguez, P., 2016. Carbon dioxide enrichment: a technique to mitigate the negative effects of salinity on the productivity of high value tomatoes. Spanish Journal of Agricultural Research 14(2), 14, doi: http://dx.doi.org/10.5424/sjar/2016142-8392.

Sánchez-Guerrero, M., 1999. Enriquecimiento carbónico en cultivos hortícolas bajo invernadero de polietileno. Universidad de Murcia.

Stanghellini, C., Incrocci, L., Gázquez, J.C., Dimauro, B., 2008. Carbon dioxide concentration in mediterranean greenhouses: how much lost production?, 801 ed. International Society for Horticultural Science (ISHS), Leuven, Belgium, pp. 1541-1550.

Valera, D.L., Belmonte, L.J., Molina-Aiz, F.D., López, A., Camacho, F., 2017. The greenhouses of Almería, Spain: technological analysis and profitability, 1170 ed. International Society for Horticultural Science (ISHS), Leuven, Belgium, pp. 219-226.

Villachica-León, C., J. Villachica-Llamosas, L. Villachica-llamosas and J. Bueno-Llamosas, 2018. Proceso HEVA: economía circular que integra la minería e industria con la agricultura y acuicultura. Foro Ayacucho / Perú. 